Oncology

Elsevier Editorial System(tm) for Clinical

Manuscript Draft

Manuscript Number:

Title: Preferences for toxicity monitoring of patients on abiraterone acetate plus prednisone

Article Type: Correspondence

Keywords: Abiraterone acetate; cancer; clinical oncology; prostate cancer; prednisone; metastatic castration-resistant; toxicity monitoring.

Corresponding Author: Dr. Elizabeth Jamieson, PhD

Corresponding Author's Institution: UCL School of Pharmacy

First Author: Heather Payne

Order of Authors: Heather Payne; Elizabeth Jamieson, PhD; Mark Prentice; Anne O'Connor; Ian Wong; Pinkie Chambers

Manuscript Region of Origin: UNITED KINGDOM 


\title{
Preferences for toxicity monitoring of patients on abiraterone acetate plus prednisone
}

\author{
H. Payne ${ }^{*}$ (corresponding author), L Jamieson ${ }^{* *}$, M Prentice $^{*}$, A O Connor ${ }^{*}$, I Wong $^{* *}$, P Chambers $^{* * *}$
}

*University College London Hospitals NHS Foundation Trust, London NW1 2PG, UK

**UCL School of Pharmacy / UCLH-UCL Centre for Medicines Optimisation Research and Education London, UK

*** University College London Hospitals NHS Foundation Trust/ UCLH-UCL Centre for Medicines Optimisation Research and Education, London, UK

Professor Heather Payne (corresponding author)

Department of Clinical Oncology

University College London Hospitals NHS Foundation Trust

250 Euston Road

London NW1 2PG

United Kingdom

Email: heather.payne@nhs.net

Liz Jamieson PhD

Research Associate

UCL School of Pharmacy \& UCLH-UCL Centre for Medicines Optimisation Research and Education 29-39 Brunswick Square

London WC1N 1 AX

United Kingdom

Email: e.jamieson@ucl.ac.uk

Dr Mark Prentice

Department of Clinical Oncology

University College London Hospitals NHS Foundation Trust

250 Euston Road

London NW1 2PG

United Kingdom

Email: mark.prentice1@nhs.net

Anne O'Connor

University College London Hospitals NHS Foundation Trust

Pharmacy Department

235 Euston Road

London NW1 2BU

United Kingdom

Email: anne.oconnor5@nhs.net

Professor lan Wong

UCL School of Pharmacy \& UCLH-UCL Centre for Medicines Optimisation Research and Education 29-39 Brunswick Square

London WC1N 1AX

United Kingdom

Email: i.wong@ucl.ac.uk 
Pinkie Chambers

University College London Hospitals NHS Foundation Trust/ UCLH-UCL Centre for Medicines Optimisation Research and Education

Pharmacy Department

235 Euston Road

London NW1 2BU

United Kingdom

Email: p.chambers@ucl.ac.uk 
Madam - Abiraterone acetate in combination with prednisone or prednisolone $(A A+P)$ is one of several novel oral hormone therapies that is effective in treating prostate cancer and is welltolerated. [1] Common adverse reactions include peripheral oedema, hypokalaemia and hypertension which are easily treated but require careful monitoring. [2][3] At University College London Hospital (UCLH) this involves additional hospital visits, twice every two weeks for the first 3 months and thereafter twice every 4 weeks in addition to routine clinic appointments for prostatespecific antigen checks and consultations.

As these extra appointments may be difficult for patients who do not live near the hospital or have other commitments, we conducted a survey to explore the preferences of patients and their carers for alternative methods of toxicity monitoring (blood testing, weight, blood pressure) and tablet collection in various settings (UCLH, GP surgery, community pharmacy) with diverse healthcare professionals.

For patients with experience of $A A+P$ this was a service evaluation and, for those with no experience of $A A+P$, it was a hypothetical exploration of their preferences

82 surveys were completed (62 patients; 20 carers). Preferences varied, indicating an individualised approach may be required. A greater percentage of carers compared to patients opted for monitoring closer to home [Table 1]. (Fisher's Exact Test 6.931, p=0.025).

Table 1 Preference for checks: Home or UCLH

\begin{tabular}{|l|c|c|c|c|c|}
\hline & \multicolumn{2}{|c|}{ Patient } & \multicolumn{2}{c|}{ Carer } & Total \\
\hline & $\mathbf{n}$ & $\mathbf{\%}$ & $\mathbf{n}$ & $\mathbf{\%}$ & \\
\hline Carried out near home & 19 & 35 & 13 & 72 & $32(44 \%)$ \\
\hline Carried out at UCLH & 26 & 48 & 4 & 22 & $30(42 \%)$ \\
\hline No real preference & 9 & 17 & 1 & 6 & $10(14 \%)$ \\
\hline TOTAL* & 54 & 100 & 18 & 100 & 72 \\
\hline
\end{tabular}

(*excluding missing data)

Those who lived further away or had other responsibilities were in favour of monitoring closer to home with the proviso that results would be communicated to UCLH. Those who had experience of $A A+P$ monitoring at $U C L H$ were satisfied/very satisfied with the service. However, alternative models including nurse specialists and telephone monitoring were acceptable alternatives for some. Many patients were prepared to monitor their weight and blood pressure and enter these into an app. Collection of tablets from local community pharmacies was strongly preferred. Alternative models would require a full evaluation. 


\section{Acknowledgements}

The SELECT project group is a Joint Working Initiative between University College London Hospitals NHS Foundation Trust, UCLH Cancer Collaborative, and Janssen-Cilag Ltd.

\section{References}

[1] Ryan CJ, Smith MR, de Bono JS, Molina A, Logothetis CJ, de Souza, P. et al. Abiraterone in metastatic prostate cancer without previous chemotherapy. N Engl J Med. 2013; 368:138-48.

[2] De Bono, JS, Logothetis CJ, Molina A, Fizazi K, North S, Chu, L. et al. Arbiraterone and Increased Survival in Metastatic Prostate Cancer N Engl J Med 2011; 364:1995-2005.

[3] Janssen-Cilag Ltd (2017). Summary of Product Characteristics: Abiraterone 500mg tablets, Electronic Medicines Compendium [online].

Available at: https://www.medicines.org.uk/emc/product/2381 [Accessed 9 April 2018] 


\section{Declarations of interest}

Heather Payne has attended and received honoraria for advisory boards, travel expenses to medical meetings and served as a consultant for AstraZeneca, Astellas, Janssen, Sanofi Aventis, Takeda, Amgen, Ipsen, Ferring, Sandoz, Roche and Novartis.

Pinkie Chambers, Ian Wong, Heather Payne and Mark Prentice have received an educational grant from Janssen unrelated to this project.

lan Wong and Pinkie Chambers have received funding from the pharmaceutical industry for research projects unrelated to this project.

Anne O'Connor was sponsored by Tessaro October 2017 to attend the British Oncology Pharmacists Association Conference. 